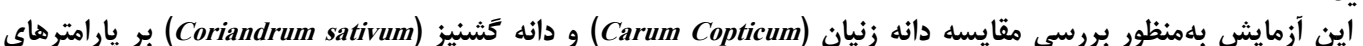

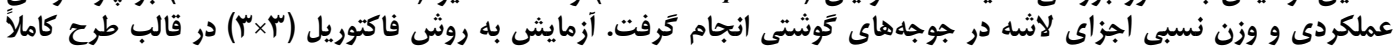

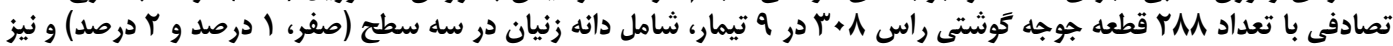

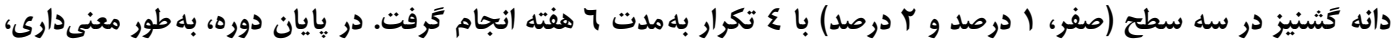

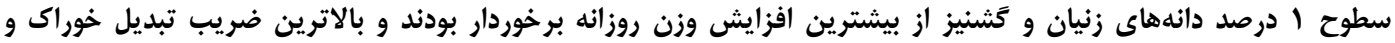

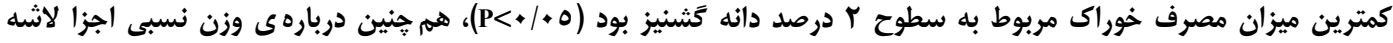

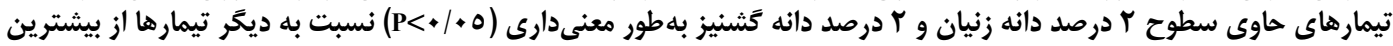

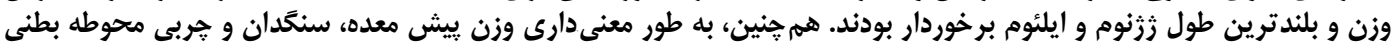

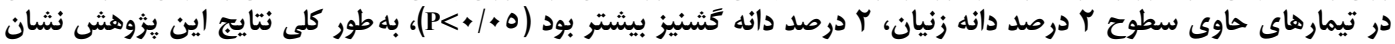

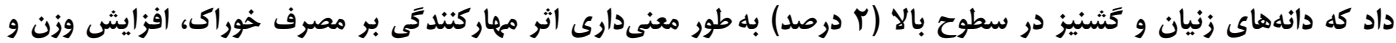

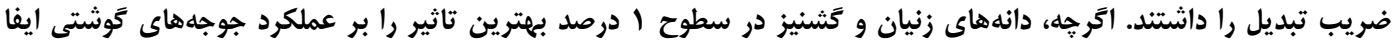
كردند.

وازههاى كليدى: دانه زنيان، دانه كَشنيز، صفات لاشه.

مىشوند. اثرات كَياهان دارويى به تركيبات شيميايى موجود در

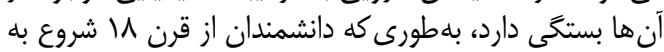

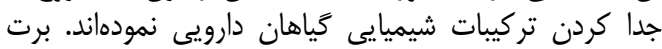

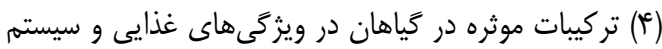

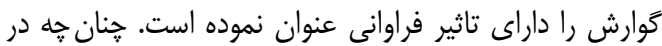

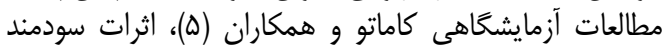

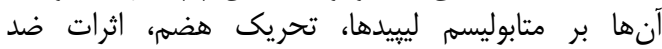

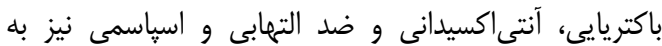
اثبات رسيده است.

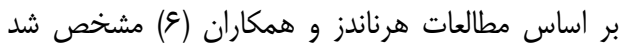

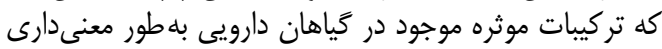

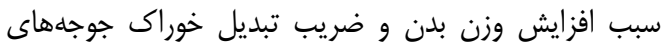

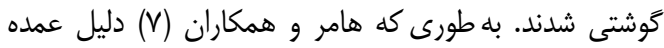

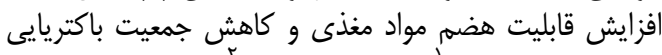

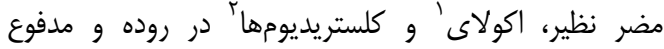

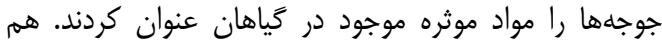

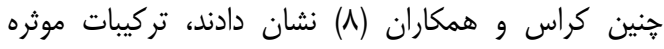

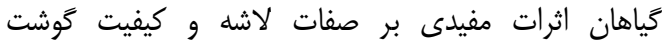
داشته|ند.

در اين باره، ارتاس و همكاران (9) و وينديش و همكاران

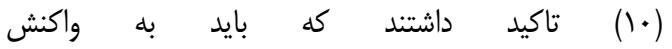
جوجههاى گوشتى به مصرف تركيبات گياهى به سطح مورد

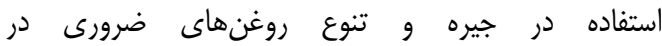
آن ها توجه داشت. موضوعى كه به نظر مى رسرهد در وهله اول 1- E.coli

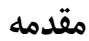

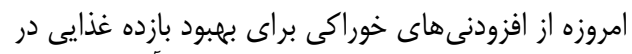

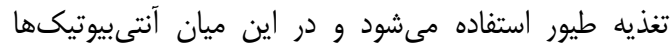

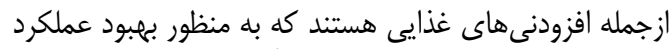

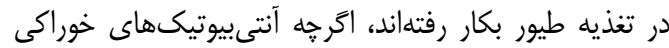

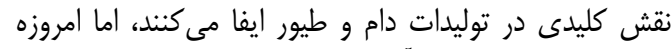

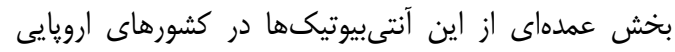

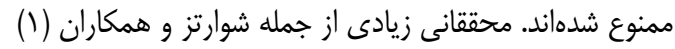

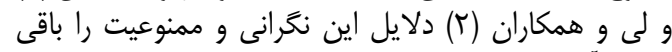

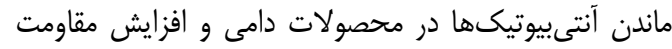

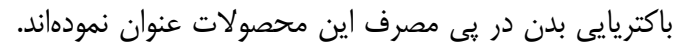

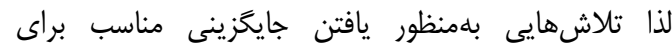

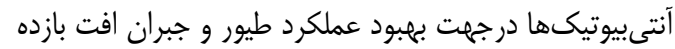

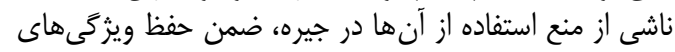

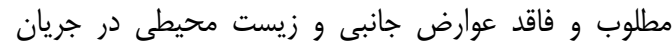

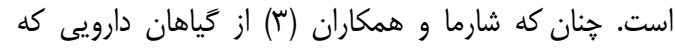
بهترين جايخزينى است كه داراى خصوصيات مذكارن مور است نام بردهاند.

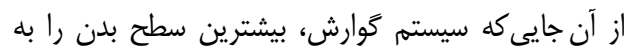

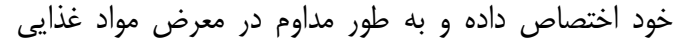

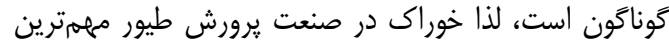

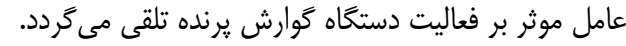

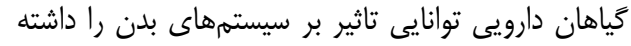

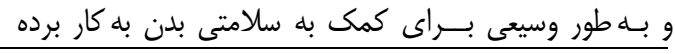




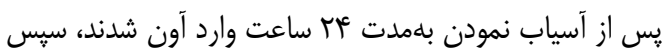

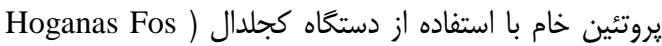

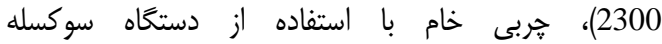

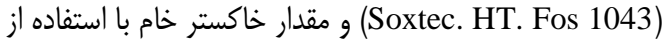

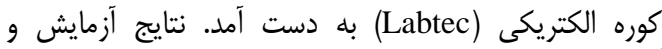

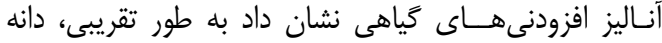

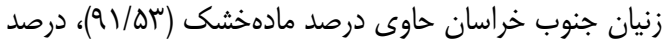

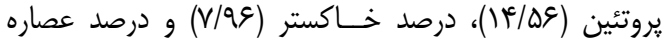

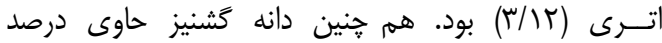

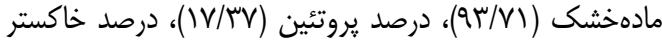

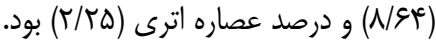

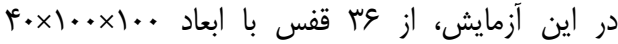

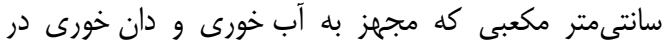

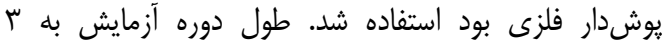

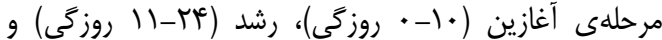

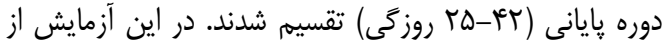

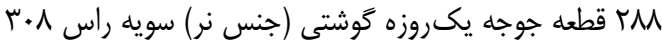

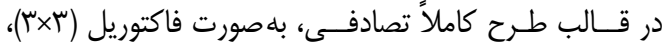

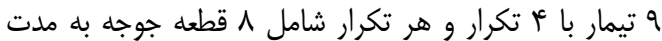

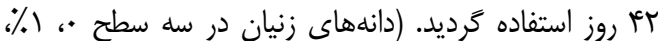

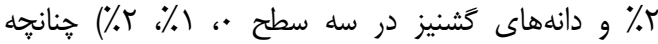
تيمارهاى آزمايشى به شرح زير بودئ إنديز

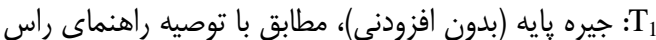
$r \cdot \Lambda$ (T:

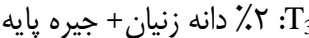
T:

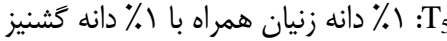

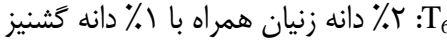

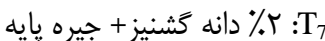
T T:

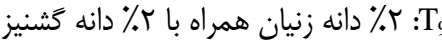

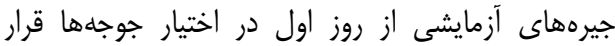

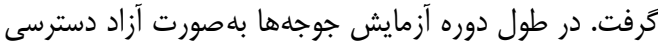

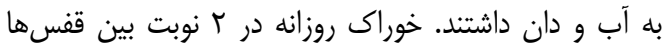

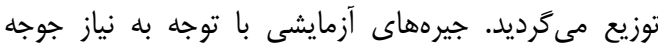

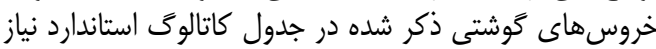

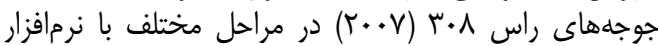
UFFDA

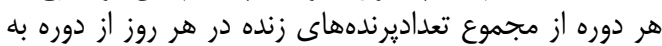

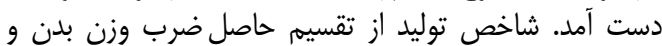

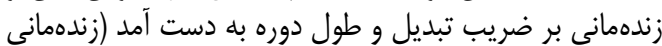

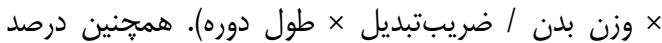

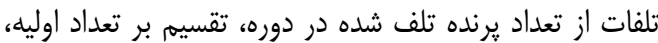

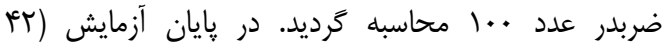

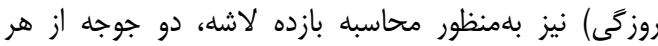

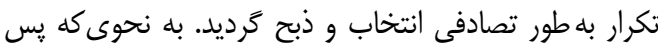

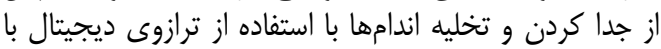

از مهمترين عوامل استفاده از مكملهاى كَاهى است. زنيان

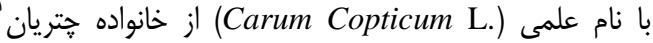

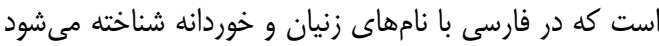

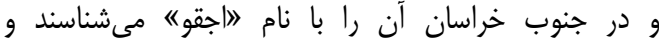

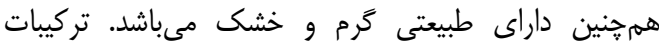

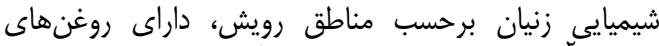

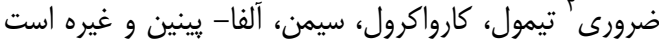

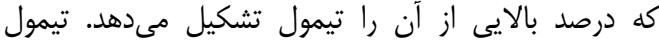

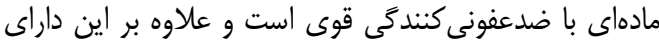
بوى معطر و طعم تندى است. بسكابادى و همكاران (11)

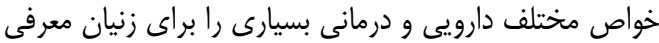

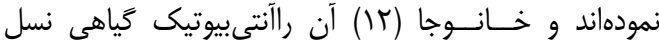
جهارم ناميده است.

كشنيز با نام علمى (Coriandrum Sativum L.)

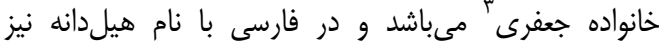

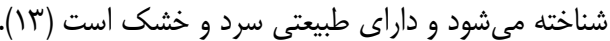

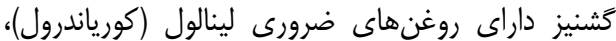

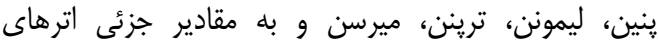
ليناليتيك است. از ديخر تركيبات فعال در ديني

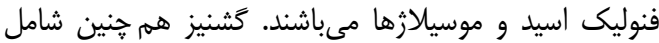

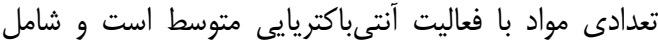
مقادير اندكى كومارين است كه خاصيت رقاريت رقيق كنندكى خون

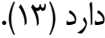

روغنهاى ضرورى تركيبات ييجِيده و فعالى هستند كه ساختار شيميايى و خواص متفاوتى دارند. اين تركيبات بر بر درين

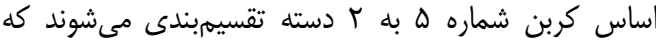

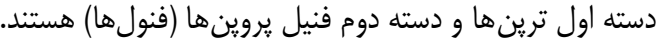

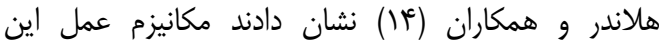
تركيبات تخريب نمودن ديواره لييويروتئينى باكترىهاست نهاست كهاني

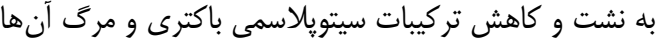

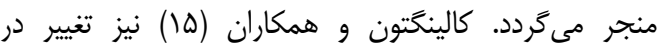

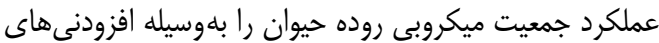

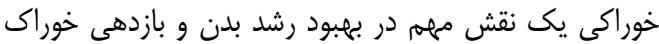

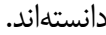

بدينمنظور، هدف از انجام اين آزمايش بررسى جُخَّكَى

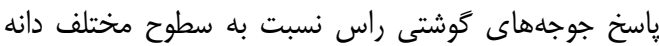

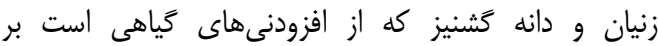
يارامترهاى عملكردى و خصوصيات لاشه آنهان آنها مىباشد.

\section{مواد و روشها}

اين أزمايش در سالن تحقيقاتى مـــرغدارى واحد دام دام

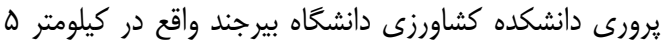

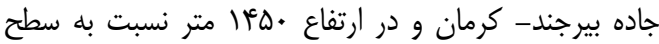

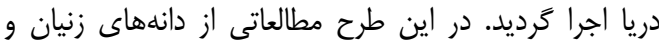

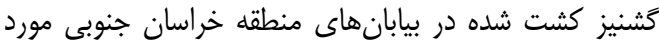

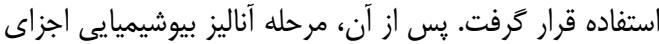

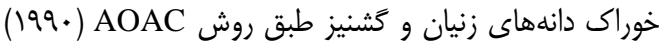

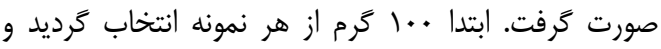


r..

از مدل آمارى آناليز دادهاى طرح فاكتوريل نيز به صورت زير بله كار گرفته شد: آمارى آنيز

$Y_{i j k l}=+Z_{j}+G_{k}+\left(Z_{j} \times G_{k}\right)+e_{i j k l}$

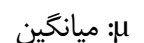
: اثر سطوح زينين

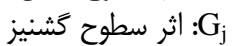
اثر : $Z_{i} \times G_{j}$ e

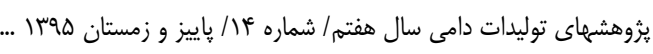

دقت 1 • /. گرم توزين شدند و وزن نسبى اين اندامها وزن و

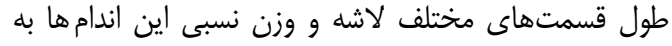

روش زير محاسبه گرديد:

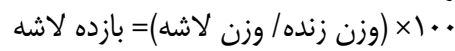

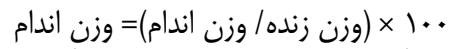

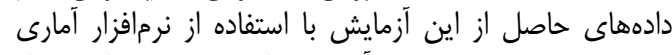

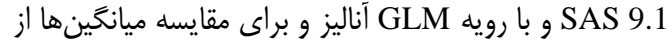

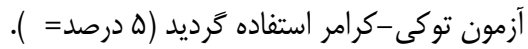

Table 1. Compostion of the experimental starter diet

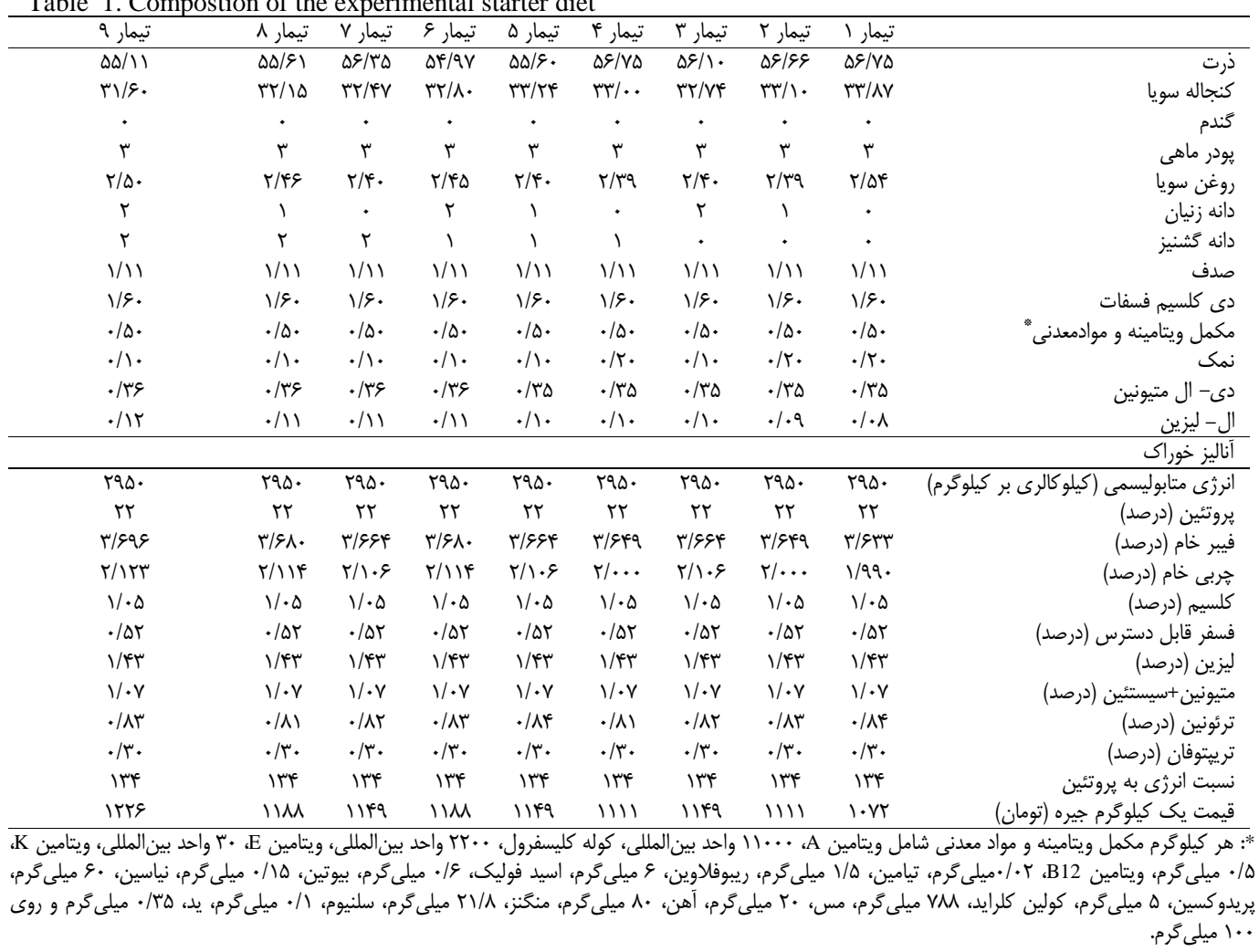


Table 2. Compostion of the experimental grower diet

جدول r- اجزاء و تركيبات جيره رشد بر حسب درصد

\begin{tabular}{|c|c|c|c|c|c|c|c|c|c|}
\hline تيمار 9 & تيمار 1 & تيمار V V & تيمار 9 & تيمار ه & تيمار f & تيمار r & Tيمار T & تيمار & \\
\hline$\Delta \backslash / V T$ & Q)/AV & $\Delta) / \Delta F$ & $\Delta I / A r$ & $\Delta / / \Delta q$ & $\Delta T / \cdot G$ & $\Delta 1 / 5 \Delta$ & D)/9V & $\Delta T / q V$ & ذرت \\
\hline$r \varepsilon|\xi|$ & $\mathrm{rV} / \mathrm{MI}$ & TN/TN & $r V / 19$ & TN/TL & TN/Nr & $r N / F q$ & rq/.. & rq/11 & كنجاله سويا \\
\hline 1. & 1. & 1. & 1. & 1. & 1. & 1. & 1. & 1. & كُندم \\
\hline r & r & $r$ & r & r & r & r & $r$ & r & يودر ماهي \\
\hline$r / \cdot V$ & Tאוא & $r / F q$ & $r / 4$. & $r / \& q$ & $r / \Delta \Delta$ & $r / F q$ & $r / r V$ & $r / \Delta S$ & روغن سويا \\
\hline r & 1 & . & r & 1 & . & r & 1 & . & دانه زنيان \\
\hline r & r & r & 1 & 1 & 1 & . & - & - & دانه گشنيز \\
\hline $1 / \cdot F^{2}$ & $1 / \cdot 4$ & $1 / \cdot f^{2}$ & $1 / \cdot f^{2}$ & $1 / \cdot f^{2}$ & $1 / \cdot f^{f}$ & $1 / \cdot \varphi^{4}$ & $1 / \cdot f^{2}$ & $1 / \cdot f^{f}$ & صدف \\
\hline $1 / 4 \wedge$ & $1 / \mp \wedge$ & $1 / 4 \wedge$ & $1 / 4 \wedge$ & $1 / 4 \wedge$ & $1 / \leftarrow \wedge$ & $1 / \mp \wedge$ & $1 / 4 \wedge$ & $1 / \uparrow \wedge$ & دى كلسيم فسفات \\
\hline.$/ 0$ & $\cdot / \Delta$ & $\cdot / \Delta$ &.$/ 0$ & $\cdot / \Delta$ & $\cdot / \Delta$ & $\cdot / \Delta$ & $\cdot / \Delta$ & $\cdot / 0$ & مكمل ويتامينه و موادمعدنى * \\
\hline$\cdot / 1$ & $\cdot$ • & $\cdot /$ & .11 & $\cdot /$ & $\cdot / 4$. & $\cdot 1$ & $\cdot / r$ & $\cdot / r$. & نمك \\
\hline.$/ 4 q$ & . & $\cdot / 4 \Lambda$ &.$/ 4 q$ & $\cdot / r \Lambda$ & $\cdot / T \Lambda$ & $\cdot / r \Lambda$ & $\cdot / 4 \Lambda$ & $\cdot / r \Lambda$ & دى- ال متيونين \\
\hline \multirow[t]{2}{*}{.$/ 19$} & $\cdot / \Lambda$ &.$/ \mathrm{V}$ &.$/ 11$ &.$/ \mathrm{V}$ &.$/ 19$ &.$/ \mathrm{VV}$ &.$/ 19$ &.$/ 19$ & ال- ليزين \\
\hline & & & & & & & & & أناليز خوراك \\
\hline$r \ldots$ & $r \ldots$ & $r \ldots$ & $r \ldots$ & $r \ldots$ & $r \ldots$ & $r \ldots$ & $r \ldots$ & $r \ldots$ & (كيلوكالرى برابركيلوكرم) \\
\hline r. & r. & r. & r. & r. & r. & r. & r. & r. & يروتئين (درصد) \\
\hline$f / \mu l$. & p/Tqf & $r / T V q$ & f/TqF & $F / T V q$ & F/TET & $F / T V q$ & F/TET & $F / T F V$ & فيبر خام (درصد) \\
\hline T/I\&q & $r / \backslash \& \mid$ & $r / T Q H$ & $r /|9|$ & T/MQT & T/NFD & $r / N Q T$ & T/NFD & $r / I r V$ & جربى خام (درصد) \\
\hline.$/ 9$ &.$/ 9$ &.$/ 9$ & $\cdot / 9$ & $\cdot / 9$ &.$/ 9$ &.$/ 9$ &.$/ 9$ &.$/ 9$ & كلسيم (درصد) \\
\hline$\cdot / \uparrow \Delta$ & $\cdot / \& \Delta$ & $\cdot / \uparrow \Delta$ &.$/ 4 \Delta$ & $\cdot / \& \Delta$ & $\cdot / \uparrow a$ & $\cdot / \mp \Delta$ & $\cdot / \uparrow \Delta$ & $\cdot / \uparrow a$ & فسفر قابل دسترس \\
\hline $1 / r$ & $1 / r$. & $1 / \tau$ & $1 / r$. & $1 / 4$. & $1 / 4$. & $1 / r$. & $1 / \tau$. & $1 / r$. & ليزين (درصد) \\
\hline.$/ 9 \Delta$ & $\cdot / 9 \Delta$ &.$/ 9 \Delta$ &.$/ 9 \Delta$ & $\cdot / 9 \Delta$ & $\cdot / 9 \Delta$ &.$/ 9 \Delta$ &.$/ 9 \Delta$ &.$/ 90$ & متيونين+سيستئين \\
\hline.$/ \mathrm{Vt}$ & $\cdot / v^{2}$ & $\cdot / N$ & $\cdot / \mathrm{V}^{\mathrm{c}}$ & $\cdot / V^{e}$ & $\cdot / V^{c}$ & $\cdot / V^{e}$ & $\cdot / V^{e}$ & $\cdot / V^{4}$ & ترئونين (درصد) \\
\hline$\cdot / T V$ & $\cdot / r V$ & $\cdot / 4 \Lambda$ & $\cdot / T V$ & $\cdot / r \Lambda$ & $\cdot / T \Lambda$ & $\cdot / r \Lambda$ & $\cdot / 4 \Lambda$ & $\cdot / r \Lambda$ & ترييتوفان (درصد) \\
\hline 10 . & 10 . & 10 . & 10 . & 10 . & 10 . & 10 . & 10. & 10. & نسبت انرزى به يروتئين \\
\hline 1191 & שr & 1149 & $1 \cdot 14$ & $1 . \wedge \kappa^{2}$ & 1.49 & $1.1 f$ & 1.49 & $1 \cdots v$ & قيمت يك كيلوگرم جيره (تومان) \\
\hline
\end{tabular}

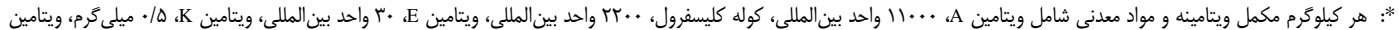

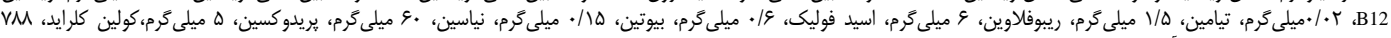

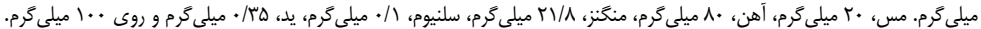

Table 3. Compostion of the experimental finisher diet

جدول س- اجزاء و تركيبات جيره يايانى بر حسب درصد

\begin{tabular}{|c|c|c|c|c|c|c|c|c|c|}
\hline تيمار 9 & تيمار 1 & تيمار V V & تيمار 9 & تيمار ه & تيمار f & تيمار r & تيمار r & تيمار 1 & \\
\hline$\Delta T / 1 \cdot$ & $01 / 98$ & $\Delta T / \cdot 1$ & $\Delta I / A V$ & $\Delta T / 19$ & $\Delta T / M Y$ & $\Delta) / A r$ & $01 / \mathrm{Vq}$ & $\Delta T / \cdot \Delta$ & ذرت \\
\hline TI/AT & rT/QT & $r r / \wedge r$ & $r \mu / \cdot r$ & Tr/g̨ & $r f / f$. & $r F / \cdot 1$ & $r F / q g$ & $r \Delta / v \cdot$ & كنجاله سويا \\
\hline 10 & 10 & 10 & 10 & 10 & 10 & 10 & 10 & 10 & 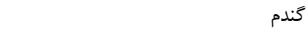 \\
\hline 1 & 1 & 1 & 1 & 1 & 1 & 1 & 1 & 1 & يودر ماهى \\
\hline$T / A V$ & T/QT & $r / \Delta V$ & $r / \Delta$. & $T / \Delta S$ & $r / \Delta \Lambda$ & $r / \Delta V$ & $r / \Delta V$ & $r / \varepsilon$. & روغن سويا \\
\hline r & 1 & • & r & 1 & - & t & 1 & • & دانه زنيان \\
\hline r & r & r & 1 & 1 & 1 & • & $\cdot$ & • & دانه كشنيز \\
\hline $1 / 4$ & $1 / .4$ & N.t & $1 / . r$ & $1 / .4$ & $1 / 2$ & D.r & I/.r & $1 / .4$ & صدف \\
\hline $1 / \Delta$ & $1 / \Delta$. & $1 / \Delta$. & $1 / \Delta$. & $1 / \Delta$ & $1 / \Delta \cdot$ & $1 / \Delta$. & $1 / \Delta$. & $1 / \Delta$ & دى كلسيم فسفات \\
\hline$\cdot / \Delta$ & $\cdot / \Delta$ & $\cdot / \Delta$ & $\cdot / \Delta$ & $\cdot 10$ & $\cdot / \Delta$ & $\cdot / 0$ & $\cdot / \Delta$ & $\cdot / \Delta$ & مكمل ويتامينه و موادمعدنى * \\
\hline.$/$ &.$/$ &.$/$ &.$/$ & .11 & $\cdot / r$ &.$/$ & $\cdot / 2$. & $\cdot / r$ & نمكى \\
\hline$\cdot / T \Delta$ & $\cdot / T \Delta$ & $\cdot / T \Delta$ & $\cdot / K \Delta$ &.$/ T \Delta$ & $\cdot / T \Delta$ & $\cdot / K \Delta$ & $\cdot / T \Delta$ & r & دى ال متيونين \\
\hline \multirow[t]{2}{*}{.$/ 4 F$} & . & $\cdot / 4 t$ & 吾 & . $/ 4 t$ & $\cdot \mid / 1$ & . & $\cdot|/|$ & $\cdot / 4 \cdot$ & ال- ليزين \\
\hline & & & & & & & & & أناليز خوراك \\
\hline$r \cdot \Delta$. & $r \cdot \Delta \cdot$ & $r \cdot \Delta$. & $r \cdot \Delta$. & $r \cdot \Delta \cdot$ & $r \cdot \Delta \cdot$ & $r \cdot \omega \cdot$ & $r \cdot \Delta \cdot$ & $r \cdot \Delta \cdot$ & انرزى متابوليسمى (كيلوكالرى بر كيلوگرم) \\
\hline$N / \Delta$ & $N / \Delta$ & $N / \Delta$ & $N / \Delta$ & $N / \Delta$ & $N / \Delta$ & $N / \Delta$ & $N / \Delta$ & $N / \Delta$ & يروتئين (درصد) \\
\hline F/ETD & $r / 9 \cdot 1$ & r/DQT & $4 / 4 \cdot \Lambda$ & r/DQT & $r / \Delta V V$ & r/DQT & $r / \Delta V$ & $F / \Delta \& 1$ & فيبر خام (درصد) \\
\hline T/TIV & $r / T \cdot q$ & $t / t \cdot t$ & $r / r+9$ & $t / t \cdot t$ & $r / 19 \%$ & $t / t \cdot t$ & $r / 194$ & $r / \wedge \Lambda \Delta$ & جربى خام (درصد) \\
\hline . / A $\Delta$ & $\cdot / \Lambda \Delta$ & $\cdot / \Lambda \Delta$ & •/AD & $\cdot / \Lambda \Delta$ & $\cdot / \wedge \Delta$ & $\cdot / \Lambda \Delta$ & $\cdot / \Lambda \Delta$ & . / A $\Delta$ & كلسيم (درصد) \\
\hline . AT & . At & . At & . & . At & . & . & . & . AT & فسفر قابل دسترس (درصد) \\
\hline $1 / \cdot 9$ & $1 / \cdot 9$ & $1 / .9$ & $1 / .9$ & $1 / .9$ & $1 / \cdot 9$ & $1 / .9$ & $1 / \cdot 9$ & $1 / \cdot 9$ & ليزين (درصد) \\
\hline . & •/A & . & •/A & $\cdot / \Lambda \varepsilon$ & • & . & $\cdot / N E$ & . & متيونين+سيستئين (درصد) \\
\hline 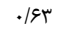 & $\cdot / 94$ & $\cdot \mid a t$ & 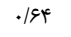 & 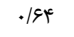 & $\cdot / 9 \Delta$ &. $\mid 94$ & $\cdot / 90$ & $\cdot / 90$ & ترئونين (درصد) \\
\hline$\cdot / 4 F$ & ./TA & $\cdot / T \Delta$ & $\cdot / T \Delta$ & $\cdot / T \Delta$ & $\cdot / T \Delta$ &.$/ T \Delta$ & $\cdot / T \Delta$ & $\cdot / T \Delta$ & ترييتوفان (درصد) \\
\hline 190 & 190 & 190 & 190 & 190 & 190 & $1 \notin 0$ & $1 \notin 0$ & 190 & نسبت انرزى به يروتئين \\
\hline $111 f$ & $1 \cdot v \varepsilon$ & $1.4 \mathrm{r}$ & $1 . v 9$ & $1 \cdot r r$ & 999 & $1 . r V$ & ११९ & १६. & قيمت يك كيلوگرم جيره (تومان) \\
\hline
\end{tabular}


باكترىهاى هوازى (كلىفرمها) در جينه دان، ثزنوم و روده

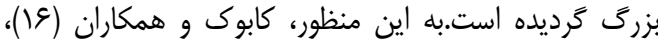

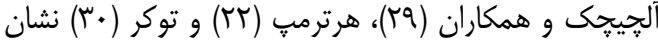

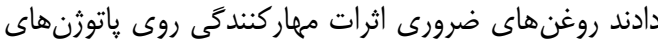

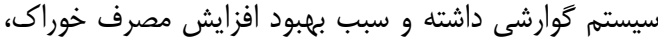

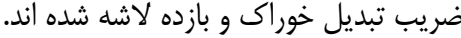

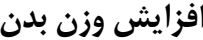
بر اساس جدول ه در دورههاى آغازين، رشد، پِايانى و

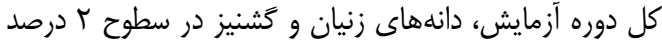

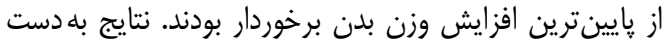

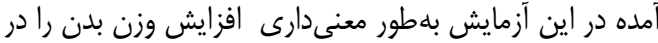

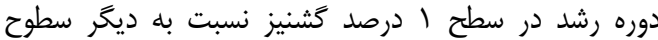

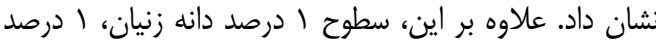

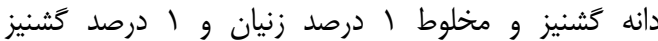

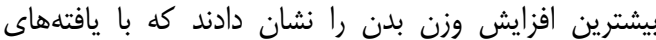

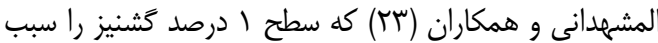

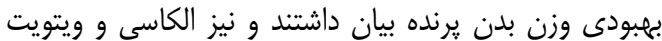

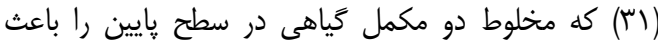

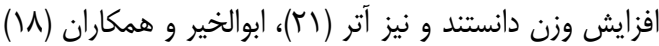

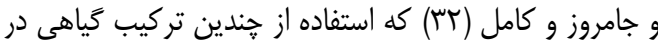

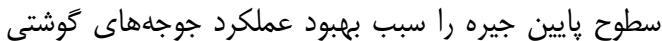

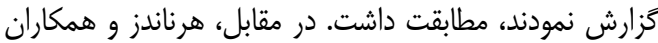

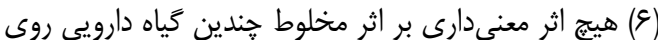

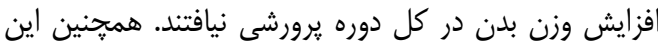

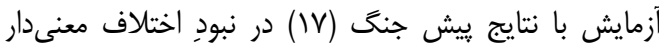

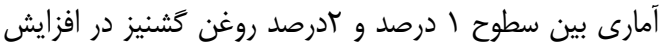

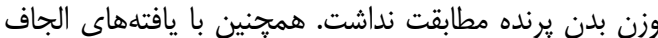

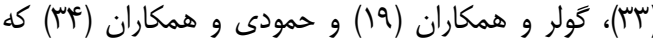

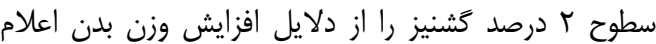
كردند، مطابقت نداشت.

اساساً، دليل عمده در كاهش وزن تيمارهاى داء حاوى سطوح

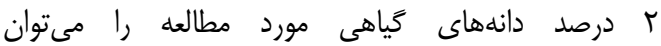
به الياف خام موجود در اين دانهها و به به دنبال آن آن افزايش

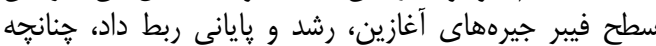

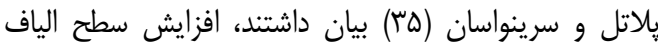
خام سبب افزايش گرانروى محتويات روده، انباشتخى رونى روده و

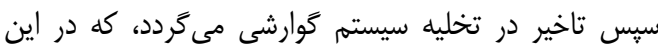

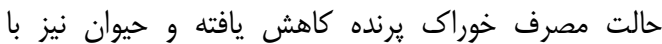

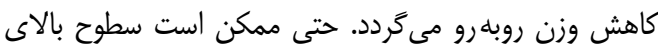

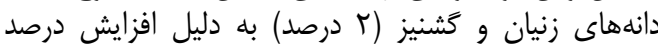

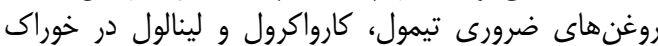

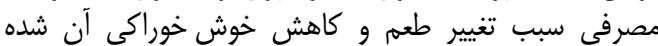

باشد.

ضريب تبديل خوراك (FCR) طبق جدول 9 بهترين ضريب تبديد تبديل خوراك در دورههاى

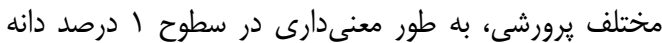

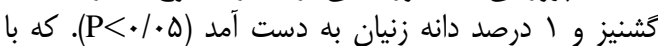

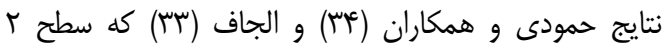

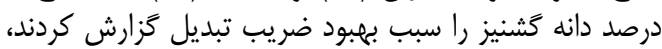

\section{نتايج و بحث يز ميزان مصرف خوراك}

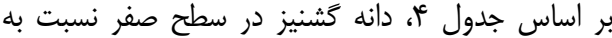

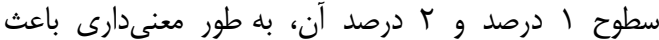

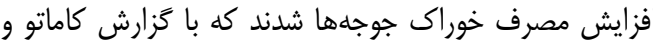

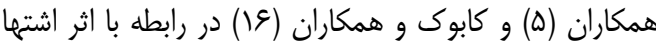
آورى كشنيز مطابقت نداشت. نتايج يزوهش

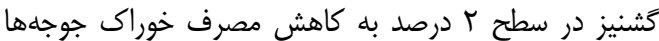

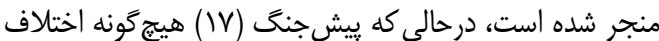

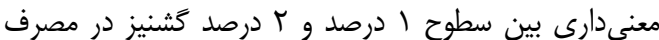

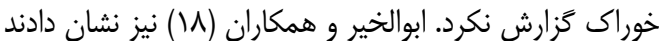

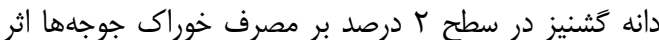

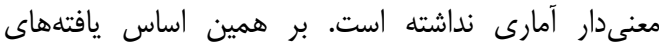

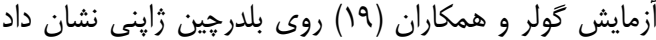

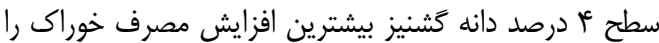

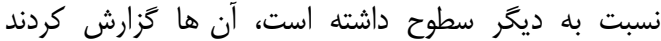

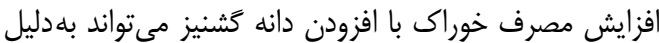

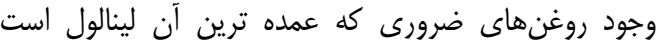

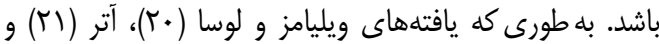

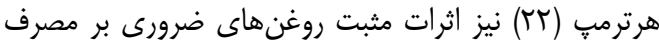

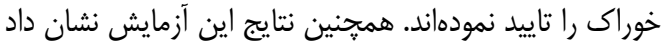

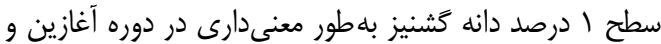

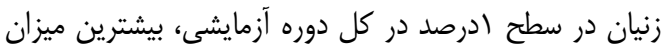

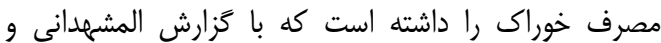

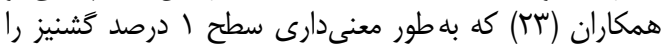

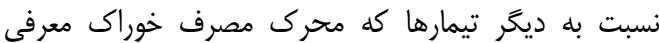

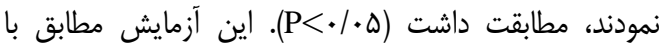

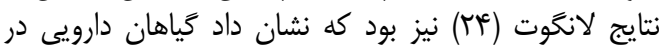

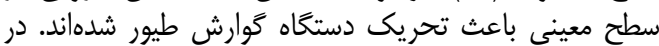

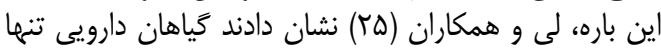

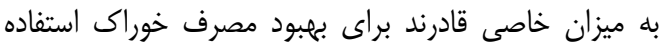

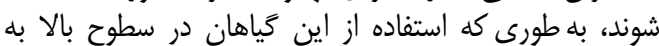

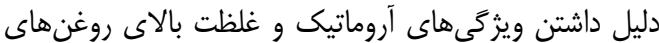

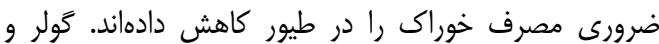

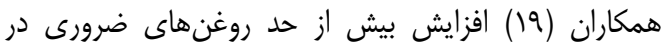

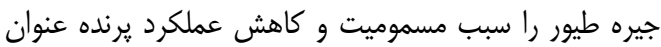

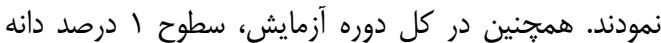

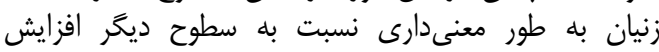

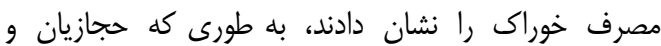

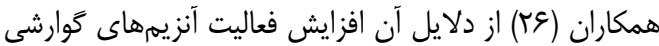

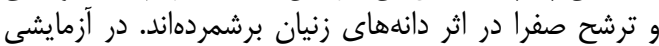

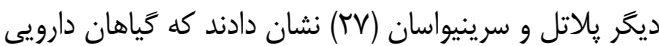

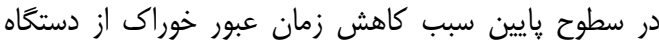

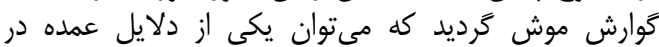

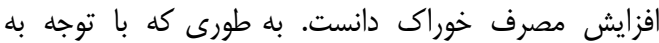

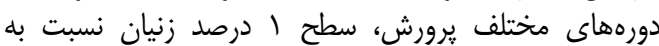

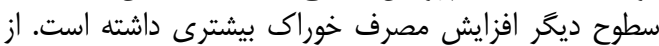

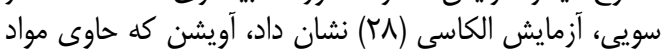
موثره تيمول و كارواكرول است، سبب كاهش آنش تعداد آنشاد 
اظهار داشتند، هنگام ورود گياهان دارويى به بدن ثيرنده،

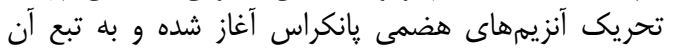

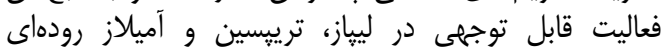

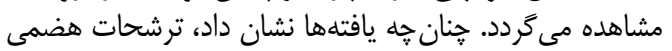

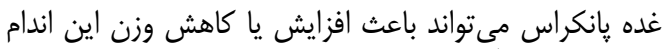

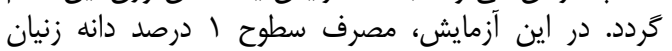

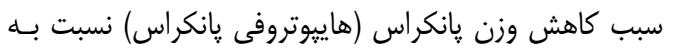

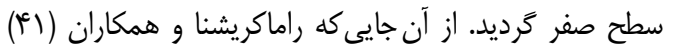

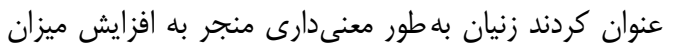

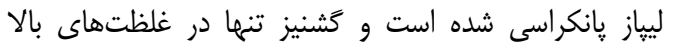

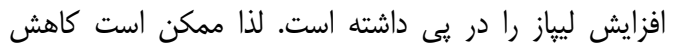

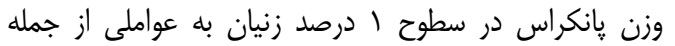

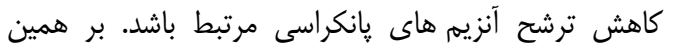

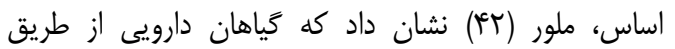

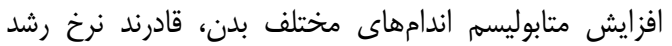

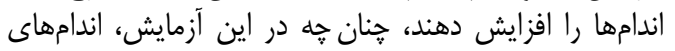

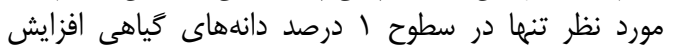

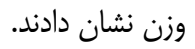

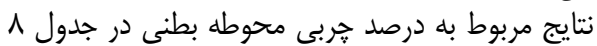

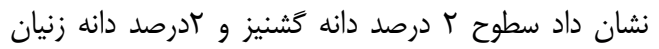

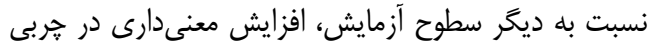

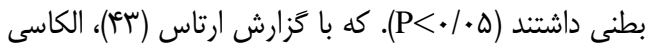

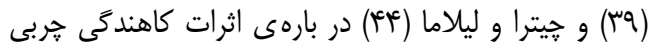

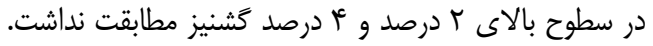

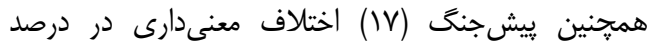

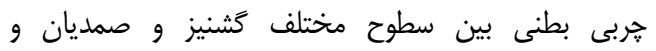

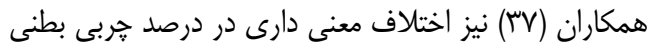

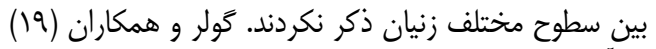

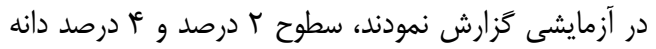

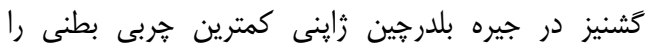

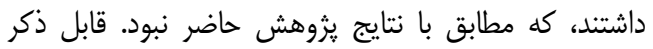

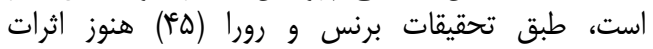
روغنهاى ضرورى بر متابوليسم ليييدها به خونى نوبى شناخته شده نيست.

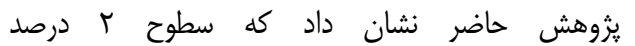

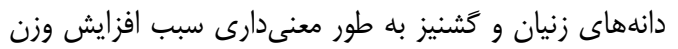

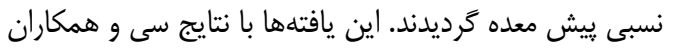

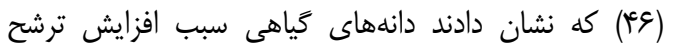

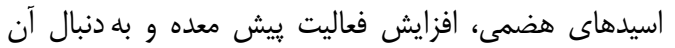

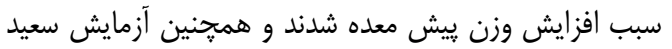

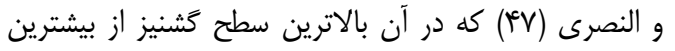

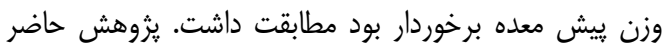

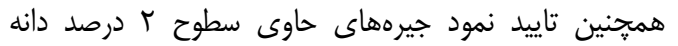

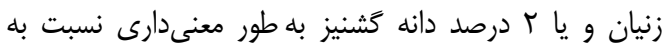

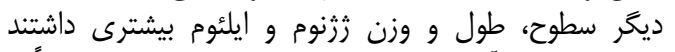

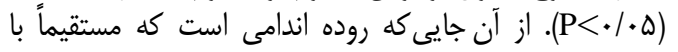

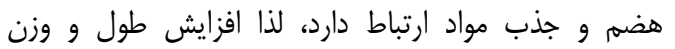

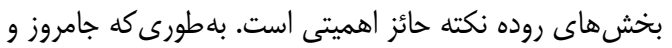

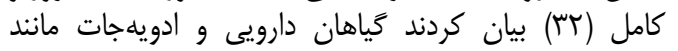

مطابقت نداشت. نتايج نشان داد سطوح ا درصد گشنيز سبب

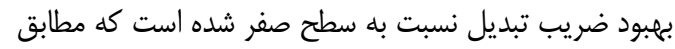

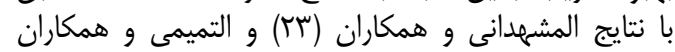

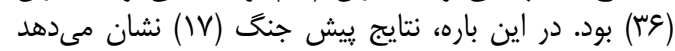

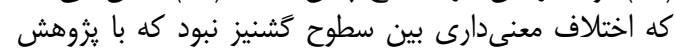

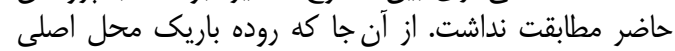

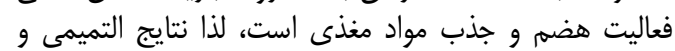

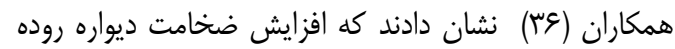

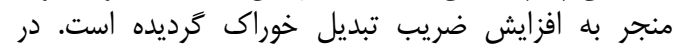

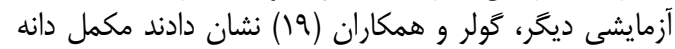

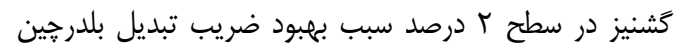

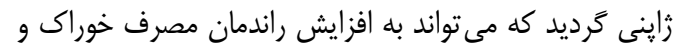

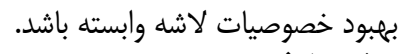

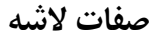

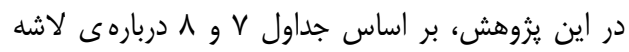

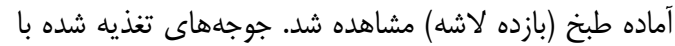

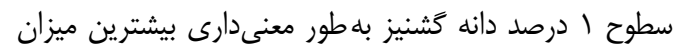

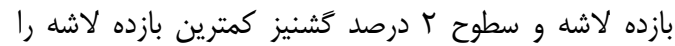

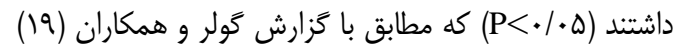

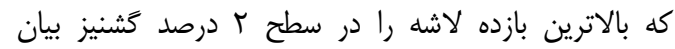

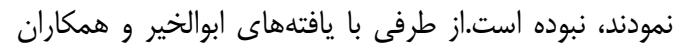

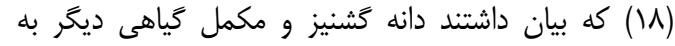

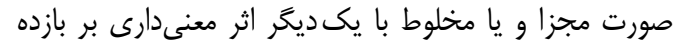

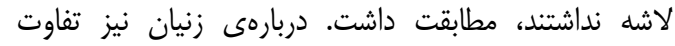

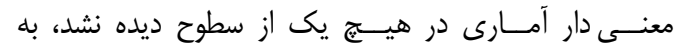

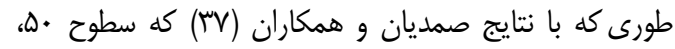

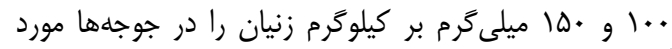

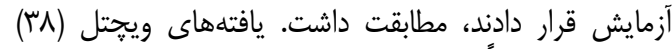

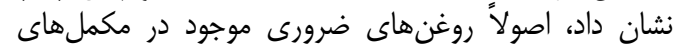

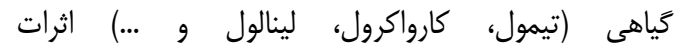

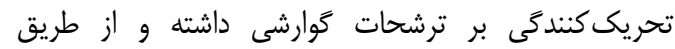

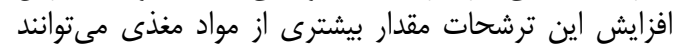

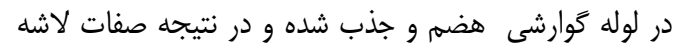

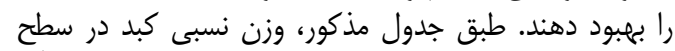

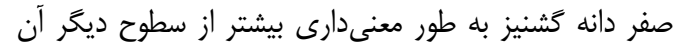

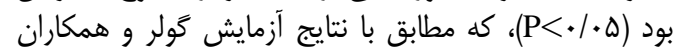

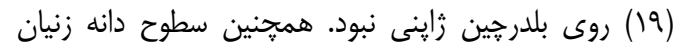

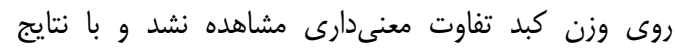

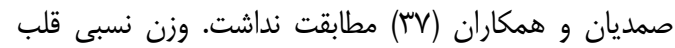

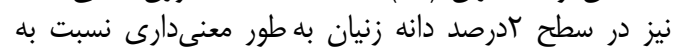

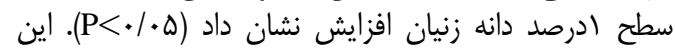

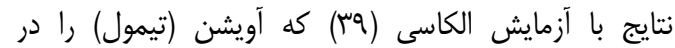

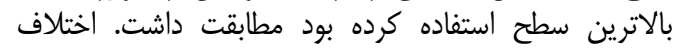

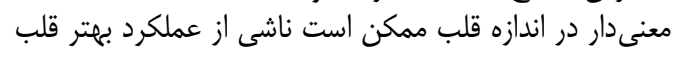

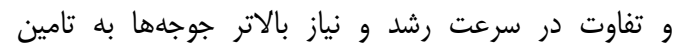

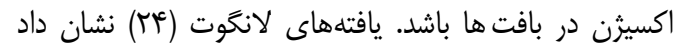

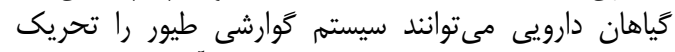

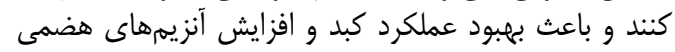

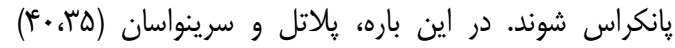




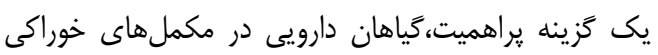

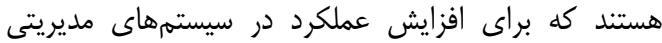

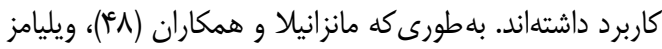

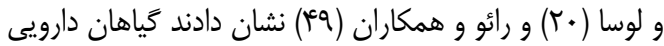

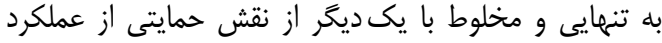

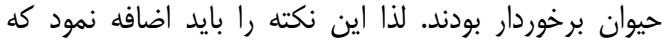

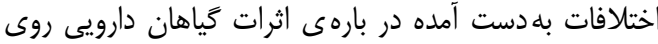

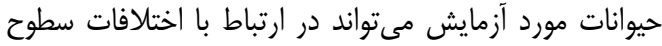

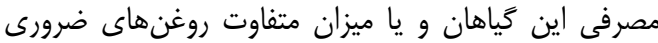

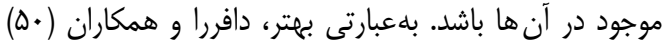

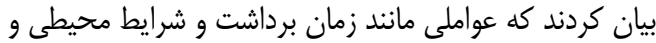

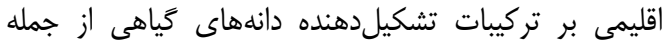

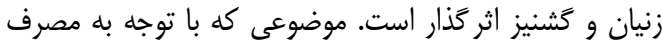

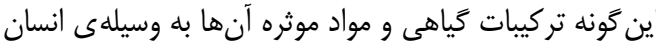

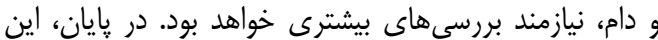

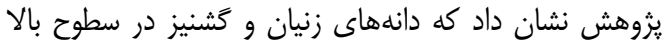

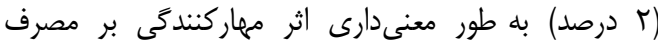

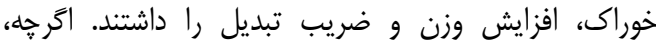

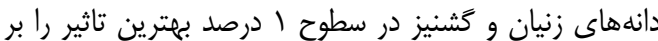

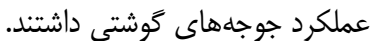

دانههاى زنيان و گثنيز قادرند باعث افزايش كرانروى

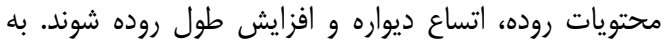

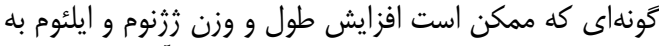

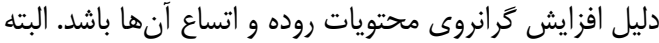

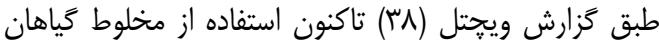

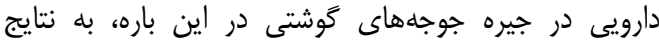
مشابهى منجر نشده است.

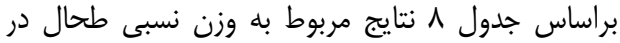

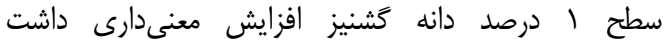

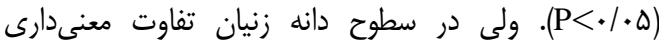

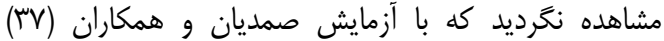

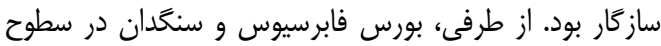

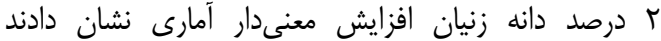

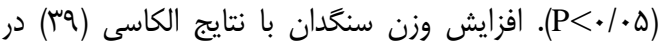

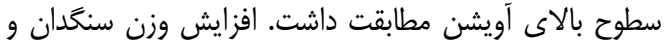

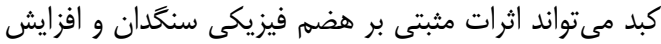

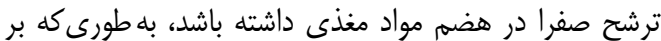

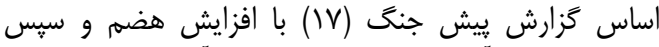
جذب اسيدهاى آمينه، رشد بدن نيز بله تبع آن افزايش مى يابد.

جدول ع- ميانگَين مصرف خوراك جوجهها در دوره رشد، يٍاينى و كل دوره آزمايش (جوجه/گرم) Table 4. The effect of different levels of Copticum and Coriander seeds on feed intake (g)

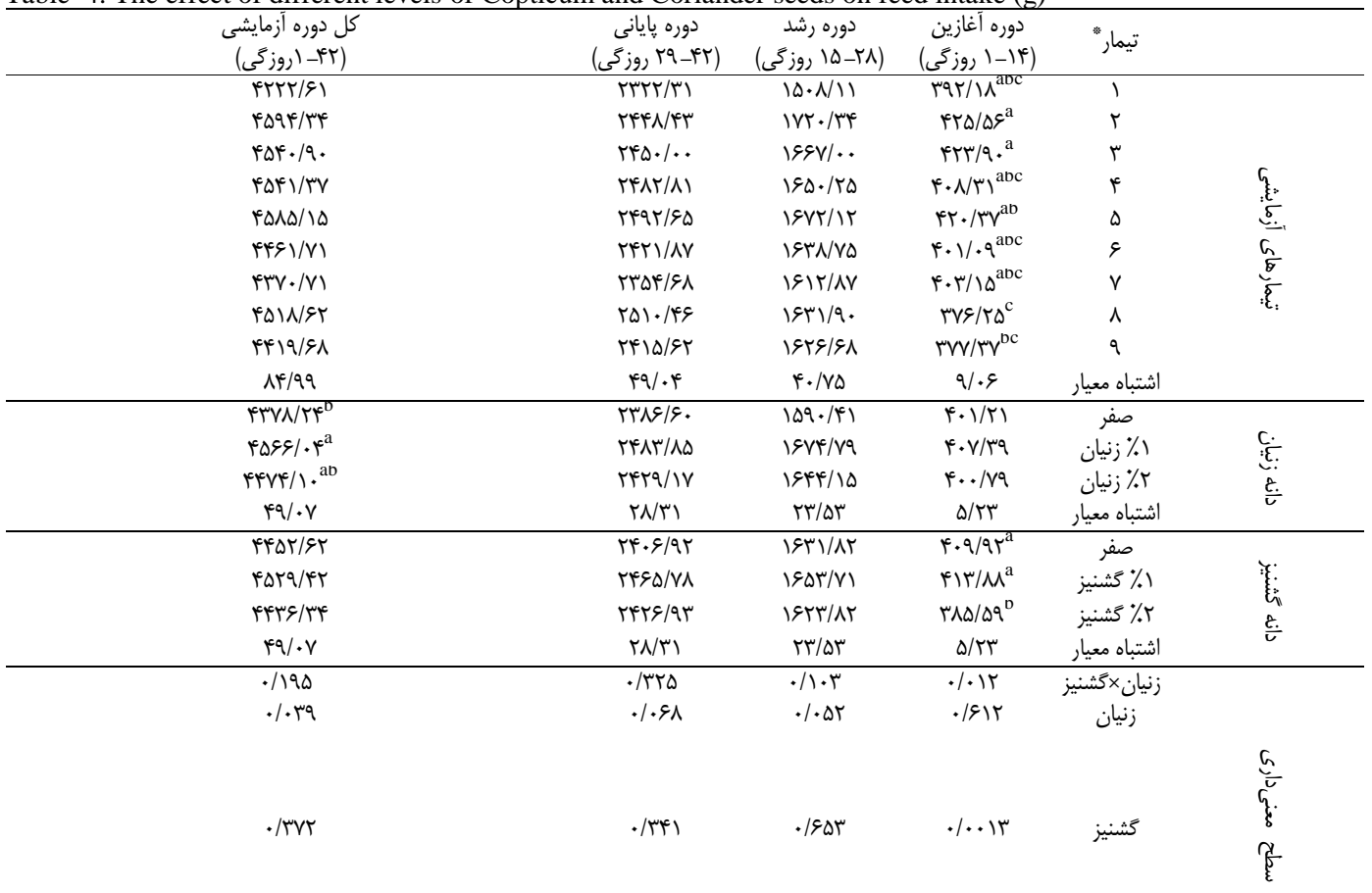


جدول ه- ميانخين افزايش وزن جوجهها در دوره رشد، پايانى و كل دوره آزمايش (جوجه/گرم) Table 5. The effect of different levels of Copticum and Coriander seeds on average weekly live weight (g)

\begin{tabular}{|c|c|c|c|c|c|}
\hline 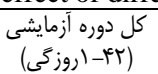 & 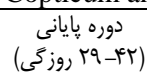 & 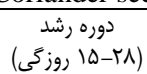 & 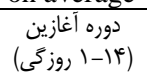 & تيمار" & \\
\hline$r \mid q q / 1 q^{a b}$ & $\mid r \cdot 1 / r \pi$ & $V \cdot N / \mp \Lambda$ & $r \Delta q / \mu r$ & 1 & \multirow{10}{*}{ 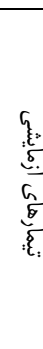 } \\
\hline TrET/qTa & $|r \varepsilon| / r$. & $V \in F / M$ & rDS/AT & r & \\
\hline$r \cdot q \vee / q^{a b c}$ & IrFq/qT & $9 \cdot 1 / v 9$ & rF\&l.. & r & \\
\hline TrFT/Fqa & $\mid r \Delta V / s$. & $\checkmark 19 / 9 \Delta$ & rED/qT & f & \\
\hline$r T \Delta q / K^{c^{a}}$ & $119 \% / \Delta 8$ & NIT/YK & rQT/qT & Q & \\
\hline $1 \Lambda \Delta F / \neg \Delta^{c}$ & $1 \cdot r \cdot / \Lambda f$ & $\Delta Q S / F V$ & שוg/RT & 8 & \\
\hline $19 \cdot 9 / \pi \omega^{\mathrm{DC}}$ & $1.94 / .4$ & $\Delta Q N / \Delta V$ & TIV/VQ & v & \\
\hline 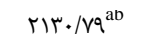 & ITEY/MT & 819/IV & $199 / r 9$ & $\wedge$ & \\
\hline${ }^{c} \mid A F T / .$. & $1.9 \% / .$. & $\Delta \Delta \& / q T$ & $19 r / \cdot V$ & 9 & \\
\hline$\Delta V / q T$ & $\Delta \cdot / \mu T$ & $r r / \Delta V$ & $V / \& D$ & اشتباه معيار & \\
\hline$r I \cdot V / \mu r^{a^{a}}$ & $11 / 4 / 99^{a 0}$ & $q V \Delta / \& V^{\circ}$ & $T Y^{e} / q V^{a}$ & صفر & \multirow{4}{*}{$\begin{array}{l}c . \\
\varepsilon \\
\varepsilon \\
\varepsilon \\
\varepsilon\end{array}$} \\
\hline$r M I V / \Lambda T^{a}$ & $\operatorname{lrm} / \mathrm{V} \cdot{ }^{\mathrm{a}}$ & $V \mp \Lambda / V v^{a}$ & مس & 1\% زنيان & \\
\hline 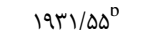 & $\| T F / \Delta Q^{D}$ & $\Delta \wedge \Delta / \cdot \Delta^{c}$ & $r \pi / 9 .{ }^{D}$ & r ز زنيان & \\
\hline ع ع/س" & $r q / \cdot \Delta$ & $\mid r / r$ & F/FT & اشتباه معيار & \\
\hline TIVG/g." & $\mid r \mu v / 4 q$ & $g \wedge \Delta / \cdot \varphi^{\mathrm{a}}$ & $r \Delta \psi / . \sigma^{a}$ & صفر & \multirow{4}{*}{$\varepsilon$} \\
\hline$r / 19 / r^{a}{ }^{a}$ & $\| \varepsilon \mid / .$. & $V \cdot q / \Delta \gamma^{a}$ & YEN/AT"a & 1\% & \\
\hline $199 \cdot / V r^{D}$ & $1145 / v q$ & $814 / 19^{\circ}$ & $r \cdot r / \cdot r^{D}$ & r \% كشنيز & \\
\hline ع צא/ & $r q / \cdot \Delta$ & $\mid r / \cdot r$ & T/ET & اشتباه معيار & \\
\hline.$/ \cdot$ rVg & $\cdot / \cdot \Delta \varphi$ &.$/ 1991$ &.$/ 4 \lambda$ & زنيان ×كَنيز & \multirow{3}{*}{ 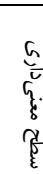 } \\
\hline $.1 \ldots 1$ & $.1 .+\Delta$ & $.1 \cdots 1$ & سו../. & زنيان & \\
\hline.$\ldots r$ & $.1 .9 \mathrm{~V}$ &. $\mid \cdots 1$ &.$|\cdots|$ & كشنيز & \\
\hline
\end{tabular}

جدول צ- ميانگين ضريب تبديل خوراك جوجهها در دوره رشد، يايانى و كل دوره آزمايش Table 6. The effect of different levels of Copticum and Coriander seeds on feed conversation ratio (g feed/g gain)

\begin{tabular}{|c|c|c|c|c|c|}
\hline 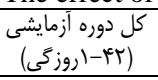 & 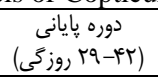 & 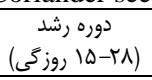 & 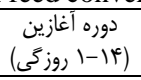 & تيمار" & \multirow{11}{*}{ 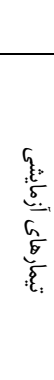 } \\
\hline $1 / 90$ & $1 / v 9$ & $1 / 9 T$ & $1 / R 9$ & 1 & \\
\hline $1 / 8 \Delta$ & $1 / v$. & $1 / 9$. & ( & r & \\
\hline $1 / \lambda$. & $1 / 81$ & $T / T F$ & $1 / \pi \Lambda$ & r & \\
\hline $1 / 4$. & $1 / 9 V$ & $1 / M$ & $1 / T r$ & $f$ & \\
\hline س & $1 / \wedge$. & I/VQ & $1 / \mu r$ & $\Delta$ & \\
\hline $1 / 94$ & $r / .9$ & ع צ/T & $1 / 49$ & 8 & \\
\hline $1 / \wedge \Delta$ & $1 / A f$ & G/T & $1 / 48$ & v & \\
\hline $1 / N$ & $1 / V T$ & $1 / 98$ & $1 / \Delta$. & $\wedge$ & \\
\hline $1 / 98$ & $1 / M$ & $r / \% Q$ & $1 / \Delta \Delta$ & 9 & \\
\hline.$/ . k T$ & $.1 \cdot \mathrm{Vr}$ &.$/ \cdot \Lambda \Delta$ &.$/ . r r$ & اشتباه معيار & \\
\hline $1 / v \cdot^{0}$ & $1 / V \Delta$ & $r / \cdot r^{D}$ & $1 / \mu^{0}$ & صفر & \multirow{4}{*}{$\begin{array}{l}c . \\
c_{0} \\
\varepsilon \\
\varepsilon\end{array}$} \\
\hline $1 / 9 \mathrm{~V}^{\mathrm{D}}$ & $1 / V^{c}$ & $1 / A v^{D}$ & $1 / \kappa^{a}$ & 1\% زنيان & \\
\hline $1 / 9 .^{a}$ & $1 / M$ & $r / \mu \Lambda^{a}$ & $1 / \kappa^{a}{ }^{a}$ & r٪ زنيان & \\
\hline.$/ . r F$ & .1 .45 & .1 .19 &.$/ .11$ & اشتباه معيار & \\
\hline $1 / \mathrm{V} \cdot{ }^{\mathrm{D}}$ & $1 / N 1$ & $r / \cdot \Delta^{a b}$ & $1 / \mu^{0}$ & صفر & \multirow{4}{*}{ 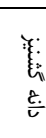 } \\
\hline $1 / V r^{D}$ & $1 / A c$ & $r / .{ }^{D}$ & $1 / r^{D}$ & 1 & \\
\hline $1 / \wedge \omega^{a}$ & $1 / A 1$ & $r / T r^{\mathrm{a}}$ & $1 / \Delta \cdot{ }^{a}$ & r \% كشنيز & \\
\hline.$/ . r F$ & $.1 .4 \mathrm{~kg}$ & $.1 .+9$ & .1 .11 & اشتباه معيار & \\
\hline .1 .949 & $\cdot / \cdot \Delta V r$ & $\cdot /$ rNAI & $\cdot \mid \Lambda \cdot \Delta Y$ & زنيان ×ثشنيز & \\
\hline.$|\ldots|$ & $.1 . v 99$ &.$|\cdots|$ &.$\ldots r$ & زنيان & $\frac{G}{\sigma}$ \\
\hline$\% \cdots \Delta$ & . $/ M T M$ & $\cdot / \cdot v V$ &.$|\cdots|$ & كشنيز & $\tilde{F}$ \\
\hline
\end{tabular}


جدول V- ميانگين وزن نسبى اجزاء لاشه (بر حسب وزن اجزاء لاشه بر وزن زنده)

Table 7. Effect of Copticum and Coriander seeds on carcass characteristics of broilers (6 weeks)

\begin{tabular}{|c|c|c|c|c|c|c|c|}
\hline بورس فابر سيوس & قلب & كبد & پانكراس & طحال & 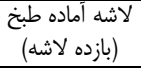 & تيمارها & \\
\hline$\cdot / T+$. & $\cdot / \Delta V^{a}{ }^{a}$ & $1 / 99^{a}$ & $\cdot / T I V^{a b}$ & $\cdot|/|^{a}$ & $\Delta T / \Delta)$ & $T$ & \\
\hline.$/ 199$ & $\cdot|\Delta r|^{\text {ad }}$ & $1 / v^{a b}$ & $\cdot / \pi / f^{\mathrm{ab}}$ & $.1 .98 \cdot{ }^{\mathrm{D}}$ & 9.194 & $r$ & \\
\hline . /TAT & $\cdot|\Delta \Delta|^{\mathrm{a}}$ & $1 / v \Delta^{a b}$ & $\cdot / r \Delta q^{a}$ & $\cdot / \cdot 0^{\mathrm{ab}}$ & $\Delta q / V$. & r & $\xi$ \\
\hline מזה & $\cdot / \Delta \cdot \gamma^{\mathrm{ab}}$ & $1 / v \cdot \Lambda^{D}$ & arava & $.1 .9 r^{\mathrm{D}}$ & س & f & : \\
\hline.$/ T F A$ & . /Qrrad & $1 / V^{a b}$ & ${ }^{\mathrm{D}} \cdot / \mathrm{A \Delta}$ & $.1 .9 \wedge \mathrm{v}^{\mathrm{ab}}$ & gF/rr & $\Delta$ & a \\
\hline (T\&9 &.$/ \Delta \varphi^{a}$ & $1 / v \cdot 9^{D}$ & $\cdot / r \cdot q^{\text {ab }}$ & $. / .99 .^{\mathrm{D}}$ & $G H / \Delta F$ & c & \\
\hline אسז/. &.$/ \Delta T F^{a d}$ & $1 / 94^{D}$ &.$/ T \Delta r^{a}$ & $.1 .940^{\mathrm{D}}$ & $\Delta N / q G$ & v & \\
\hline$\cdot / r \cdot$. & $\cdot / F \Delta F^{D}$ & $1 / \Delta V^{D}$ & ${ }^{a b} \cdot / r T \Delta$ & $.1 .99 .^{\mathrm{D}}$ & rr/M & $\wedge$ & \\
\hline . /TNA & $\cdot / \Delta \Delta \Lambda^{\mathrm{a}}$ & $1 / \wedge r^{a b}$ & ${ }^{a b} \cdot / \pi / Q$ & $.1 .94 \hat{c}^{\mathrm{D}}$ & $p q / q 4$ & 9 & \\
\hline (1/. & .1 .19 &.$/ \cdot \Delta \Delta$ & I... & 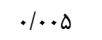 & $r / q V \mid$ & اشتباه معيار & \\
\hline$\cdot / r \Lambda^{0}$ & $\cdot / \Delta r c^{a D}$ & I/VAr & $\cdot / r \mu r^{a}$ & $\cdot / 1 \cdot V 9$ & $\Delta N / \mu$. & صفر & \\
\hline.$/ r I V^{D}$ & $\cdot(\Delta \cdot)^{0}$ & $1 / v \cdot 1$ & $\cdot / 4 \cdot 1^{D}$ & $\cdot N \cdot r V$ & gr/AD & $\%$ & \\
\hline 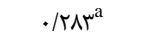 & $\cdot|\Delta s|^{\mathrm{a}}$ & I/VEY & $\cdot / r r \Lambda^{a b}$ & $\cdot 11 \cdots \infty$ & $\Delta V / V r$ & $\%$ & 4 \\
\hline $.1 . .9$ & אזו/. & D & $.1 . .9$ &.$/ \cdot r V$ & $1 / v r r$ & اشتباه معيار & \\
\hline . /KFV & $\cdot / \Delta \Delta f^{F}$ & $1 / \lambda \uparrow \Lambda^{a}$ & $\cdot$ & $.1 .1111^{\circ}$ & $\Delta N / \cdot \leqslant 9^{0}$ & صفر & \\
\hline . /TQT & TH & $1 / R r^{D}$ & $\cdot / r \cdot 9$ & $\cdot / N \cdot r \cdot{ }^{a}$ & $\varepsilon \mu / F r \Delta^{a}$ & $\% 1$ & \\
\hline qם & . $/ \Delta I T$ & $1 / 8 \wedge \Gamma^{D}$ & . & $1.9 V \Delta^{\mathrm{ab}}$ & $\Delta V / F^{C} \cdot 9^{D}$ & $\%$ & c \\
\hline $.1 . .9$ &.$/ \cdot 1 r \Lambda$ & 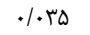 & $.1 . .9$ & q & $1 / A / f$ & اشتباه معيار & \\
\hline$\cdot / 1 \cdot \Lambda V$ &.$/ . F A Y$ &.$/ .148$ & .1 .199 &.$/ \cdot 1$ & $\cdot / \cdot \mathrm{VA}$. & زنيان×5شنيز & \\
\hline. $\mid \cdots+1$ & $\cdot / \cdot v^{c}$ & $\cdot / 4 \cdot 19$ & |. & ./TهQ1 & $\cdot / \cdot N \Delta F$ & زنيان & \\
\hline$\cdot 19 \cdot 4 q$ & $\cdot / \cdot \mathrm{rr}$ &...+4 & $\cdot 1 \cdot 0 \cdot 4$ & $\cdot / \cdot r \cdot \Lambda$ & $.1 \cdot$ erf & كشنيز & \\
\hline
\end{tabular}

*: ميانكَين هاى هر ستون با حروف غيرمشتر داراى اختلاف معنىدار مىباشند.

جدول ^- ميانخين وزن نسبى اجزاء لاشه (بر حسب وزن اجزاء لاشه بر وزن زنده) Table 8. Effect of Copticum and Coriander seeds on carcass characteristics of broilers (6 weeks)

\begin{tabular}{|c|c|c|c|c|c|c|c|}
\hline وزن روده & طول ايلئوم & طول زثنوم & ييشمعده & سنگحدان & جربى محوطه بطنى & تيمارها & \multirow{11}{*}{ 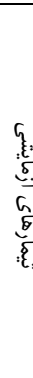 } \\
\hline מא/א & $\mathrm{rr} / \mathrm{Aq}^{\mathrm{abc}}$ & $\Gamma \mathrm{T} / 9 \Delta^{\mathrm{abc}}$ & $\cdot / H F \cdot{ }^{c}$ & $1 / V r^{D C}$ & $\cdot / \mathrm{V}^{\mathrm{Cd}}$ & 1 & \\
\hline$r / \Delta F$ & $\mu F / q^{a b c}$ & 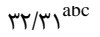 &.$|\& \&|^{a b}$ & $1 / \mathcal{A r}^{\mathrm{DC}}$ & $\cdot \mid \Delta \xi^{c a}$ & r & \\
\hline r/AV & $r q / 19^{\text {ad }}$ & $\Gamma \Delta / \kappa^{\mu} \gamma^{a b c}$ & $\cdot 10 \cdot 9^{a}$ & $1 / 99^{a}$ & $1 / \pi r^{a}$ & r & \\
\hline سז/ץ & $r / / Y^{\mathrm{Dc}}$ & $T \mathcal{H} / \Delta \mathcal{F}^{\mathrm{abc}}$ & $\cdot / f y \cdot \cdot^{a b}$ & $1 / \wedge \varepsilon^{a}$ & $\cdot / v \Lambda^{\mathrm{e}}$ & f & \\
\hline$r / f V$ & $r \cdot / v \cdot^{c}$ & $r q / q r^{c}$ & Dc & $1 / M^{\mathrm{Dc}}$ & $\cdot / V \Delta^{\mathrm{ca}}$ & $\Delta$ & \\
\hline$r / v r$ & $r r / \cdot 1^{\mathrm{DC}}$ & $r \cdot / q \Psi^{\mathrm{Dc}}$ &.$/ 4 \& q^{D c}$ & $1 / v^{a b c}$ & $\cdot / \Lambda \Delta^{\mathrm{ca}}$ & 9 & \\
\hline$r / \Delta q$ & $r \Delta / 1 q^{a b c}$ & $|g / \Delta|^{\text {ab }}$ & $\cdot / 4 \gamma \cdot \cdot^{a}$ & $1 / 1 q^{a}$ & $.119^{0}$ & $\checkmark$ & \\
\hline$m / f 1$ & $r / / q \Lambda^{\mathrm{dc}}$ & $\Psi \backslash / / f^{a b c}$ & $\cdot / T \Delta q^{D c}$ & $1 / f \Delta^{c}$ & $\cdot / \Lambda r^{\mathrm{ae}}$ & $\wedge$ & \\
\hline$r / . r$ & $\Gamma \Lambda / \Gamma \omega^{a}$ & $r / v^{a} q^{a}$ &.$/$ prra $^{\mathrm{a}}$ & $1 / 9 \Delta^{\mathrm{a}}$ &.$/ 9 v^{a}$ & 9 & \\
\hline.$/ 1 F \wedge$ & I/TM & I/TVT & $.1 \cdot 10$ & $\cdot 1 \cdot \mathrm{nl}$ & سץ./. & اشتباه معيار & \\
\hline$r / F T \cdot{ }^{D C}$ & $r / T \Psi^{\mathrm{ad}}$ & $\Gamma / \Gamma \Delta \Delta^{D}$ &.$/ 418^{\mathrm{aD}}$ & $\mid / \wedge I^{D}$ & $\cdot / \Lambda \mid r^{D}$ & صفر & \multirow{4}{*}{$\begin{array}{l}c . \\
\qquad \\
\varepsilon \\
\varepsilon\end{array}$} \\
\hline$\Gamma / F V \Delta^{c}$ & $r / T \cdot 9^{D}$ & r/৭१ & $\cdot / L^{e} \cdot r^{D}$ & $1 / 4 \ldots .^{c}$ & . & $\%$ & \\
\hline $\mathrm{r} / \Lambda V^{\mathrm{a}}$ & $r / \mathcal{} r r^{a}$ & & $\cdot / \& \Delta \cdot \cdot^{a}$ & $1 / 91 r^{\mathrm{ab}}$ & ./9ه سa & $\%$ & \\
\hline $.1 .9 \mathrm{~V}$ & .1 .90 & .1 .941 & $\cdot 1 \cdot 1+$ & 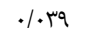 & $\cdot / 411$ & اشتباه معيار & \\
\hline$\Gamma / \Delta \Delta I$ & $\mathrm{~T} / \mathrm{KqV}^{\mathrm{aD}}$ & $r / M M^{a b}$ & $\cdot / 4 r f^{a 0}$ & I/VNS & $\cdot / \Lambda \uparrow \varepsilon^{D}$ & صفر & \\
\hline W/DF & $r / 19 \gamma^{D}$ & $r / \cdot 19^{0}$ & $\cdot / \mathrm{m}^{\mathrm{c}} \mathrm{c}$ & 1/vq.. & $\cdot / V r^{c}$ & $\%$ & \\
\hline$r / g V$ & $r / \& q)^{a}$ & a سזس/ץ &.$/ F V^{D}$ & I/VEV & . $/ \wedge 98^{a b}$ & $\%$ & \\
\hline$\cdot|\cdot 1|$ & .1 .90 & $.1 .9 \mathrm{VI}$ & $.1 .+9$ & $.1+41$ &.$/ 411$ & اشتباه معيار & \\
\hline . & $\cdot / \cdots \wedge 1$ &.$/ \cdot T \Delta D$ &.$/ \cdots \cdot 1$ & $\cdot / \cdot \Delta r$ & $.1+\cdots 1$ & زنيان ×گشنيز & \multirow{3}{*}{ 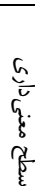 } \\
\hline$. / \cdots+1$ & $.1 \cdot 1 V f$ & $\cdot 1+\cdots 1$ & .1 tef &.$/ \cdots 1$ &.$|\cdots|$ & زنيان & \\
\hline r. rm/. & $\cdot 1 \cdots \wedge \Delta$ & $.1 \cdot 4<q$ & $.1 \cdot 1 \cdot 4$ & זr/91. & $.1 \cdots+$ & كَنيز & \\
\hline
\end{tabular}


$r \cdot$

1. Schwarz S. C. Kehrenberg and T.R. Walsh 2001. Use of antimicrobial agents in veterinary medicine and food animal Production. International Journal of Antimicrobial Agents, 17: 431-437.

2. Lee, K.W., H. Everts and A.C. Beynen. 2004. Essential oils in broiler nutrition. International Journal of Poultry Science, 3: 738-752.

3. Sharma, A., M. Kumar and S. Kaur. 2011. Cuminum cyminum L. and Coriandrum sativum L. extracts modulate Chromium geno toxicity in Allium cePa chromosomal aberration assay. Nucleus, 2: 99-105.

4. Burt, S. 2004. Essential oils: their antibacterial ProPerties and Potential aPPlications in foods, a review. International Journal of Food Microbiology, 94: 223-253.

5. Kamatou, G.P.P., N.P. Makunga, W.P.N. Ramogola and A.M. Viljoen. 2008. South African salvia sPecies: A review of biological activities and Phyto chemistry. Journal of EthnoPharmacology, 119: 664-672.

6. Hernandez, F., J. Madrid, V. Garcia, J. Orengo and M.D. Megias. 2004. Influence of tow Plant extract on broiler Performance, digestibility, and digestive organ size. Poultry Science, 83: 169-174.

7. Hammer, K.A., C.F. Carson and T.V. Riley. 1999. Antimicrobial activity of essential oils and other Plant extracts. Journal of APPlied Microbiology, 86: 985-990.

8. Cross, D.E., R.M. Mcdevitt, K. Hillman and T. Acamovic. 2007. The effects of herbs and their associated essential oils on Performance, dietary digestibility and gut microflora in chickens from 7 to 28 days of age, British Poultry Science, 48: 496-506.

9. Ertas, O., N. Guler and U.G. Simsek. 2005. The effect of an essential oil mix derived from oregano, clove and anise on broiler Performance. International Journal of Poultry Science, 4: 879-884.

10. Windisch, W., K. Schedle, C. Plitzner and A. Kroismayr. 2008. A use of Phytogenic Products as feed additives for swine and Poultry. Journal of Animal Science, 86: 140-148.

11. Boskabady, M.H., S. Alitaneh and A. Alavinezhad. 2014. Carum Copticum L.: A herbal medicine with various Pharmacological effects. BioMed Research International, 2014: 1-11.

12. Khanuja, S.P.S. 2004. Formulation com Prising thymol useful in the treatment of drug resistance bacterial infection. CCIR, New Delhi (India), United State Patent.

13. Zargari, A. 1992. Plant Medicines. 6rd edn., Tehran University Press, Tehran, Iran, PP: 900. (In Persian).

14. Helander, I.M., K.S. Latva-Kala and K. Lounatmaa. 1998. Permeabilizing action of Polyethyleneimine on Salmonella tyPhimurium involves disruPtion of the outer membrane and interactions with liPoPolysaccharide. Microbiology, 144: 385-390.

15. Collington, G.K., D.S. Parker and D.G. Armstrong. 1990. The influence on inclusion of either an antibiotic or Probiotic in the diet on the develoPment of digestive enzyme activity in the Pig. British Journal Nutrition, 64: 59-70.

16. Cabuk, M.A., M. Alcicek, N. Bozkurt and N. Imre. 2003. Isolated from aromatic Plants and using Possibility as alternative feed additives. National Animal Nutrition Congress, 18-20 September, 184-: $187 \mathrm{pp}$, Turkey.

17. Pish Jang, J. 2011. Effect of different levels of coriander oil on Performance and blood Parameters of broiler chickens. Annals of Biological Research, 2: 578-583.

18. Abou-Elkhair, R., H.A. Ahmed and S. Selim. 2014. Effects of Black PePPer (PiPer Nigrum), Turmeric Powder (Curcuma Longa) and Coriander Seeds (Coriandrum Sativum) and Their Combinations as Feed Additives on Growth Performance, Carcass Traits, Some Blood Parameters and Humoral Immune ResPonse of Broiler Chickens. Asian Australasian Journal of Animal Science, 27: 847-854.

19. Guler, T., O.N. Ertas. M. Ciftci and C.B. Dalki. 2005. The effect of coriander seed (Coriandrum sativum L.) as diet ingredient on the Performance of JaPanese quail. South African Journal of Animal Science, 35: 261-267.

20. Williams, P. and R. Losa. 2001. The use of essential oils and their comPounds in Poultry nutrition. World Poultry, 17: 14-15.

21. Ather, M.A.M. 2000. Poly herbal additive Proves effective against vertical transmission of IBD. World Poultry, 16: 50-52.

22. HertramPf, J.W. 2001. Alternative antibacterial Performance Promoters. Poultry International, 40: $50-52$.

23. Al-Mashhadani, E.H., F.K. Al-Jaff, S.J. Hamodi and H.E. Al-Mashhadani. 2011. Effect of coriander oil on broiler Performance and some Physiological traits under high ambient temPerature. Pakistan Journal of Nutrition, 10: 10-14.

24. Langhout, D.J., J.B. Schutte, J. De Jong, H. Sloetjes, M.W. Verstegen and S. Tamminga. 2000. Effect of viscosity on digestion of nutrients in conventional and germ-free chicks. British Journal of Nutrition, 83: 533-540.

25. Lee, K.W., H. Everts, H.J. KaPPert, M. Frehner, R. Losa and A.C. Beynen. 2003. Effects of dietary essential oil comPonents on growth Performance, digestive enzymes and liPid metabolism in female broiler chickens. British Poultry Science, 44: 450-457.

26. Hejazian, S., M. Morowatisharifabad and S. Mahdavi. 2007. Relaxant effect of Carum Copticum on intestinal motility in ileum of rat. World Journal of Zoology, 2: 15-18.

27. Platel, K. and K. Srinivasan. 2001. Studies on the influence of dietary sPices on food transit time in exPerimental rats. Nutrition Research, 21: 1309-1314.

28. Al-Kassie, G.A.M. 2010. The effect of thyme and cinnamon on the microbial balance in gastro intestinal tract on broiler chicks. International Journal of Poultry Science, 9: 495-498. 
29. Alçiçek, A., M. Bozkurt and M. Çabuk. 2003. The effect of essential oil combination derived from selected herbs growing wild in Turkey on broiler Performance. South African Journal of Animal Science, 33: 89-94.

30. Tucker, L. 2002. Botanical broilers: Plant extracts to maintain Poultry Performance. Feed International, 23: 26-29.

31. Al-Kassie, G.A.M. and N.M. Witwit. 2010. A comParative study on diet supplementation with a mixture of herbal Plants and dandelion as a source of Prebiotics on the Performance of broilers. Pakistan Journal of Nutrition, 9: 67-71.

32. Jamroz, D. and C. Kamel. 2002. Plant extracts enhance broiler Performance. Journal of Animal Science, 80: 140-148.

33. Al-Jaff, F.K. 2011. Effect of coriander seeds as diet ingredient on blood Parameters of broiler chicks raised under high ambient temPerature. International Journal of Poultry Science, 10: 82-86.

34. Hamodi, S.J., E.H. Al-mashhadani, F.K. Al-jaff and H.E. Al-mashhadani. 2010. Effect of coriander Seed (Coriandrum sativum L.) as diet ingredient on broilers Performance under high ambient temPerature. International Journal of Poultry Science, 10: 968-691.

35. Platel, K. and K. Srinivasan. 2000. Influence of dietary sPices and their active PrinciPles on Pancreatic digestive enzymes in albino rats. Food, 44: 41-46.

36. Al-Tememy, H.S.A., F.K. Al-Jaff, E.H. Al-Mashhadani and S.J. Hamodi. 2011. Histological effect of inclusion different levels of coriander oil in broiler diet on small intestine. Diyala Agricultural Sciences Journal, 3: 1-11.

37. Samadian, F., S. Zeinoaldindi, A. Towhidi, M.A. Torshizi, Z.A. Pirasaraei and P. Gholamzadeh. 2013. Evaluation of some Phytogenic feed additives in growing chickens diets. International Journal of Agriculture, 3: 35-43.

38. Wichtl, M. 1994. Herbal drugs and phyto pharmaceuticals crc press, Stuttgart, Germany, pp: 159-160.

39. Al-Kassie, G.A.M. 2009. Influence of two Plant extracts derived from thyme and cinnamon on broiler Performance. Pakistan Veterinary Journal, 29: 169-173.

40. Platel, K. and K. Srinivasan. 2004. Stimulant action of sPices: A myth or reality. Indian Journal of Medical Research, 119: 167-179.

41. Ramakrishna, R., K. Platel and K. Srinivasan. 2003. In vitro influence of sPices and sPice active PrinciPles on digestive enzymes of rat Pancreas and small intestine. Food Nahrung, 47: 408-412.

42. Mellor, S. 2000. Antibiotics are not the only growth Promoters. World Poultry, 16: 14-15.

43. Ertas, O.N. 2013. The effect of dietary suPPlement coriander seeds on abdominal fat dePosition and fatty acid comPosition in Japanese quail Journal of Animal and Veterinary Advances, 12: 48-51.

44. Chithra, V. and S. Leelamma. 1997. HyPoliPidemic effect of coriander seeds (Coriandrum sativum): mechanism of action. Plant Foods Humanity Nutrition, 51: 167-72.

45. Brenes, A. and E. Roura. 2010. Essential oils in Poultry nutrition: Main effects and modes of action. Animal Feed Science and Technology, 158: 1-14.

46. Si, W., J. Gong, R. Tsao, Y.H. Zhou, C. PoPPe, R. Johnson and Z. Du. 2006. Antimicrobial activity of essential oils and structurally related synthetic food additives towards selected Pathogenic and beneficial gut bacteria Journal of APPlied Microbiology, 100: 296-305.

47. Saeid, J.M. and A.S. AL-Nasry. 2010. Effect of dietary coriander seeds supplementation on growth Performance carcass traits and some blood Parameters of broiler chickens. International Journal of Poultry Science, 9: 867-870.

48. Manzanilla, E.G., M. Nofrarias, M. Anguita, M. Castillo, J.F. Perez, S.M. Martin, C. Kamel and J. Gasa. 2006. Effects of butyrate, avilamycin, and a Plant extract combination on the intestinal equilibrium of early-weaned Pigs. Journal of Animal Science, 84: 2743-2751.

49. Rao, R.R., K. Platel and K. Srinivasan. 2003. In vitro influence of sPices and sPice active PrinciPles on digestive enzymes of rat Pancreas and small intestine. Food, 47: 408-412

50. Daferera, D.J., B.N. Ziogas and M.G. Polissiou. 2000. GC-MS analysis of essential oils from Greek aromatic Plants and their fungi toxicity on Penicillum digitatum. Journal Agriculture Food Chemistry, 48: 2576-2581. 


\title{
Screening for Effects of Different Levels of Ajowan (Carum Copticum L.) and Coriander (Coriandrum Sativum L.) Seeds on Performance and Carcass Characteristics of Ross Broiler Chickens
}

\author{
Saeed Alitaneh ${ }^{1}$, Nazar Afzali ${ }^{2}$, Hadi Sarir ${ }^{3}$ and Hossein NaeimiPour ${ }^{4}$ \\ 1- Graduated M.Sc., Birjand University (Corresponding author: saeed.alitaneh@gmail.com) \\ 2 and 3- Professor and Assistant Professor, Birjand University, Iran \\ 4- Ph.D. Student, Ferdowsi University, Mashhad \\ Received: March 15, $2014 \quad$ Accepted: January 11, 2015
}

\begin{abstract}
This experiment was conducted to evaluate the effects of ajowan (Carum Copticum) and coriander (Coriandrum sativum) seeds on growth Performance and carcass characteristics. A total of 288 one-day old chickens (Ross 308$)$ were used as factorial experiment $(3 \times 3)$ in a completely randomized design in 9 treatments including ajowan levels $(0,1 \%$ and $2 \%)$ and coriander levels $(0,1 \%$ and $2 \%)$ with 4 replications and 10 birds each for 6 weeks age. Results were showed treatments containing $1 \%$ levels of ajowan and coriander seeds showed significantly better weight gain and performance compared to other treatments $(\mathrm{P}<0.05)$ whereas, the level of $2 \%$ did not show a proper Performance. High feed conversion ratio (FCR) and lowest feed intake were observed in 2 percent levels of coriander $(\mathrm{P}<0.05)$. In relation of carcass characteristics, contents 2 Percent of coriander and 2 Percent of ajowan seeds $(\mathrm{P}<0.05)$ had the most weight and longest jejunum and ileum than in the other treatments. The weight of proventriculus, gizzard and abdominal fat levels were significantly higher in 2 Percent ajowan and coriander seeds $(\mathrm{P}<0.05)$. Overall, the result of this study showed that significantly, high levels ajowan and coriander seeds $(2 \%)$ have inhibitor effect for feed intake, weight gain and FCR. However, were useful of coriander and ajowan seeds in 1 percent levels.
\end{abstract}

Keywords: Ajowan (Carum Copticum), Coriander (Coriandrum sativum), Carcass traits 